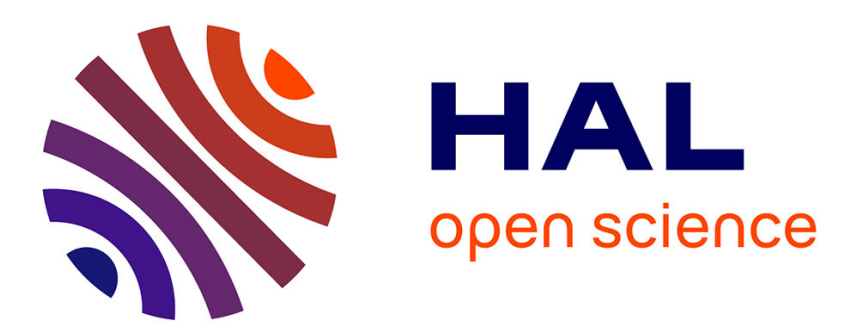

\title{
Effects of phase change on reflection in phase-measuring interference microscopy
}

Arnaud Dubois

\section{To cite this version:}

Arnaud Dubois. Effects of phase change on reflection in phase-measuring interference microscopy.

Applied optics, 2004, 43, pp.1503. hal-00533152

\section{HAL Id: hal-00533152 \\ https://hal.science/hal-00533152}

Submitted on 26 Jan 2012

HAL is a multi-disciplinary open access archive for the deposit and dissemination of scientific research documents, whether they are published or not. The documents may come from teaching and research institutions in France or abroad, or from public or private research centers.
L'archive ouverte pluridisciplinaire HAL, est destinée au dépôt et à la diffusion de documents scientifiques de niveau recherche, publiés ou non, émanant des établissements d'enseignement et de recherche français ou étrangers, des laboratoires publics ou privés. 


\title{
Effects of phase change on reflection in phase-measuring interference microscopy
}

\author{
Arnaud Dubois
}

\begin{abstract}
We show by analytical and numerical calculations that the phase change on reflection that occurs in interference microscopy is almost independent of the numerical aperture of the objective. The shift of the microscope interferogram response due to the phase change on reflection, however, increases with the numerical aperture. Measurements of the interferogram shift are made with a Linnik interference microscope equipped with various numerical-aperture objectives and are reported and compared with theory. (C) 2004 Optical Society of America
\end{abstract}

OCIS codes: $180.3170,120.5050,120.2830,120.6650,120.3180$.

\section{Introduction}

Phase-measuring interference microscopy is a powerful, widely used technique for smooth-surface profiling with micrometric and nanometric resolutions in the lateral and axial directions, respectively. ${ }^{1-3}$ When the surface to be profiled is composed of dissimilar materials, the difference in the phase change of light reflected from these different materials gives rise to height measurement offsets. ${ }^{4-6}$ This problem can be overcome by coating the surface with a thin metallic layer. This coating is, however, undesirable in most applications. Another approach involves applying compensating offset factors by the calculation of the phase change on reflection (in normal incidence). This method requires prior knowledge of the complex refractive indices of the materials. The phase change on reflection can also be measured by interferometric and microellipsometric techniques. ${ }^{7-12}$ The phase change on reflection depends on the illumination incidence angle. ${ }^{13}$ In interference microscopes the sample is illuminated with a distribution of incidence angles defined by the numerical aperture of the objective. Therefore the phase change on reflection in interference microscopy

The author (dubois@optique.espci.fr) is with Laboratoire d'Optique Physique, Ecole Supérieure de Physique et Chimie Industrielles, Centre National de la Recherche Scientifique, Unité Propre de Recherche A0005, 10 rue Vauquelin, F-75231 Paris Cedex 5 , France.

Received 14 August 2003; revised manuscript received 11 November 2003; accepted 6 November 2003

0003-6935/04/071503-05\$15.00/0

(C) 2004 Optical Society of America would be expected to depend on the numerical aperture. As already suggested by numerical simulations, the variation of the phase change on reflection with incidence angle of unpolarized light seems, however, to be weak. ${ }^{11}$

We establish in this paper an analytical expression for the phase change on reflection as a function of incidence angle. We study the effect of the phase change on reflection on the interferogram response of the interference microscope, taking into account the distribution of incidence angles through the objective. Measurements are carried out with a Linnik interference microscope with various numerical apertures.

\section{Illumination Angle Dependence of the Phase Change on Reflection}

We establish in this section an analytical expression for the phase change on reflection as a function of incidence angle for unpolarized light. We assume that the light is reflected from the surface of a planar object placed in air. The amplitudes $\left|r_{s}\right|,\left|r_{p}\right|$ and the arguments $\phi_{s}, \phi_{p}$ of the complex reflection coefficients $r_{s}$ and $r_{p}$ of the object for the $s$ - and $p$-polarization components, respectively, can be calculated as a function of incidence angle $\theta$ by use of general Fresnel equations. ${ }^{11,13}$ These quantities depend on the complex index of refraction $\hat{n}=n+i k$ of the material. The use of complex arithmetic can be avoided by the introduction of expressions involving only real quantities. These expressions are

$$
\left|r_{s}(\theta)\right|=\left(\frac{N^{2}+K^{2}-2 N \cos \theta+\cos ^{2} \theta}{N^{2}+K^{2}+2 N \cos \theta+\cos ^{2} \theta}\right)^{1 / 2},
$$




$$
\begin{aligned}
& \left|r_{p}(\theta)\right|= \\
& \left|r_{s}(\theta)\right|\left(\frac{N^{2}+K^{2}-2 N \sin \theta \tan \theta+\sin ^{2} \theta \tan ^{2} \theta}{N^{2}+K^{2}+2 N \sin \theta \tan \theta+\sin ^{2} \theta \tan ^{2} \theta}\right)^{1 / 2},
\end{aligned}
$$$$
\tan \phi_{s}(\theta)=\frac{-2 K \cos \theta}{N^{2}+K^{2}-\cos ^{2} \theta},
$$$$
\tan \phi_{p}(\theta)=\frac{2 K \cos \theta\left(N^{2}+K^{2}-\sin ^{2} \theta\right)}{N^{2}+K^{2}-\left(n^{2}+k^{2}\right)^{2} \cos ^{2} \theta},
$$

with the intermediate variables $N$ and $K$ defined as

$$
\begin{aligned}
N= & \frac{1}{2}\left\{\left[\left(n^{2}-k^{2}-\sin ^{2} \theta\right)^{2}+4 n^{2} k^{2}\right]^{1 / 2}\right. \\
& \left.+\left(n^{2}-k^{2}-\sin ^{2} \theta\right)\right\}, \\
K= & \frac{1}{2}\left\{\left(n^{2}-k^{2}-\sin ^{2} \theta\right)^{2}+4 n^{2} k^{2}\right]^{1 / 2} \\
& \left.-\left(n^{2}-k^{2}-\sin ^{2} \theta\right)\right\} .
\end{aligned}
$$

In the case of normal incidence $(\theta=0)$, Eqs. (5) and (6) become $N=n, K=k$, and Eqs. (1)-(4) simplify to well-known formulas.5,11,13 The amplitude and phase changes on reflection for both $s$ and $p$ components are coherent with respect to each other. The total phase change $\phi$ on reflection is the argument of the vector sum of the two components ${ }^{8,13}$ :

$$
\phi(\theta)=\arg \left[\left|r_{s}\right| \exp \left(i \phi_{s}\right)+\left|r_{p}\right| \exp \left(i \phi_{p}\right)\right] .
$$

Finding an explicit general expression for $\phi$ as a function of $\theta$ is not possible. Developing Eqs. (1)-(4) to order $\theta^{3}$, we obtain

$$
\begin{aligned}
\left|r_{p}(\theta)\right| & =\left|r_{s}(\theta)\right|\left[1-\gamma \theta^{2}+O\left(\theta^{4}\right)\right], \\
\phi_{s}(\theta) & =\phi(0)+\varepsilon \theta^{2}+O\left(\theta^{4}\right), \\
\phi_{p}(\theta) & =\phi(0)-\varepsilon \theta^{2}+O\left(\theta^{4}\right),
\end{aligned}
$$

where

$$
\begin{aligned}
\gamma & =\frac{2 n}{n^{2}+k^{2}} \\
\varepsilon & =\frac{k}{n^{2}+k^{2}} \\
\phi(0) & =\arctan \left[\frac{2 k}{1-\left(n^{2}+k^{2}\right)}\right] \quad(\operatorname{modulo} \pi) .
\end{aligned}
$$

The phase change in normal incidence is $\phi(0)$ (equal for $s$ and $p$ polarizations). $\varepsilon$ is the first nonzero term (second-order term) in the power expansion of $\phi_{s}(\theta)$ and $\phi_{p}(\theta) . \quad \varepsilon$ characterizes the amplitude of the phase variations with incidence for the $s$ - and $p$-polarization components. At third order the phase variations with incidence for the $s$ and $p$ components are strictly opposed. Substituting Eqs. (8)-

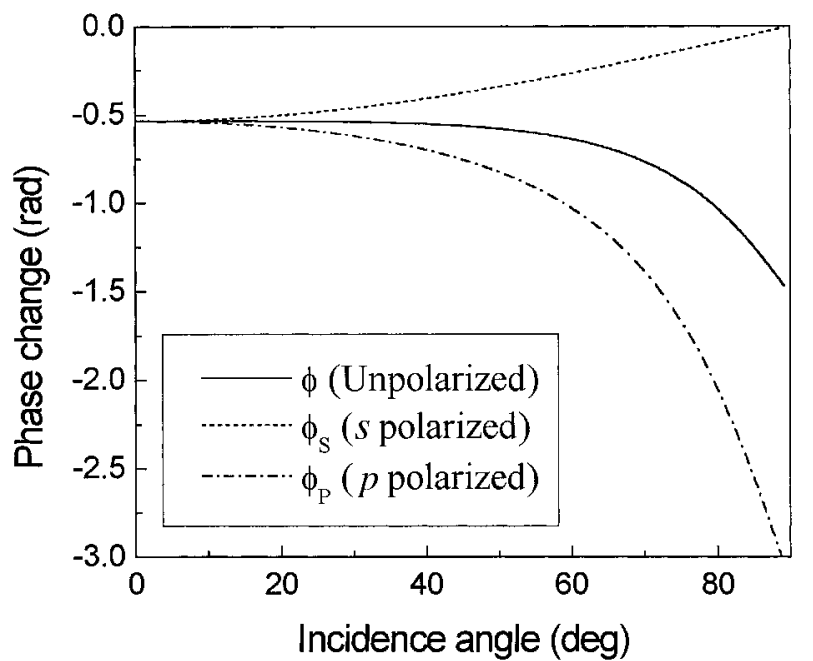

Fig. 1. Phase changes of light on reflection air and copper versus incidence angle for the $s$ - and $p$-polarization components and for unpolarized light. $\phi_{s}(\theta), \phi_{p}(\theta)$, and $\phi(\theta)$ are calculated modulo $\pi$ by use of Eq. (3), Eq. (4), and Eq. (7), respectively, at a wavelength of $650 \mathrm{~nm}$. The refractive indices of copper are $n=0.224$ and $k=$ $3.63 .{ }^{14}$

(10) into Eq. (7), we obtain the expression for the total phase change on reflection of unpolarized light to third order in incidence $\theta$ :

$$
\phi(\theta)=\phi(0)+O\left(\theta^{4}\right) .
$$

We have shown here that $\phi$ does not depend on the incidence angle at the first, second, and third orders. This result is in agreement with numerical simulations that show a weak variation of $\phi$ with $\theta$ even in the case of reflection on metals (see Fig. 1 and Ref. 11).

\section{Effect of the Phase Change on Reflection on the Interference Microscope Response}

In an interference microscope, such as the Michelson, Mirau, or Linnik, the sample is illuminated by a cone of converging rays with incidence angles $\theta$ varying from 0 to $\theta_{\max }$, where $\theta_{\max }$ is related to the numerical aperture (NA) of the objective by NA $=\sin \theta_{\max }$ (in air). ${ }^{15,16}$ Assuming aberration-free aplanetic optics and monochromatic spatially incoherent illumination at the wavelength $\lambda=1 / \sigma$, the modulated part of the interferogram response of the microscope can be written as $^{15-17}$

$$
M(z)=\int_{0}^{\theta_{\max }} \cos (4 \pi \sigma z \cos \theta+\phi(\theta)) \cos \theta \sin \theta \mathrm{d} \theta .
$$

The variable $z$ is the on-axis geometrical path difference between the object and the reference. $\phi(\theta)$ is the absolute phase change of reflected light on the object surface. The reflection on the reference surface is assumed to introduce no phase change (modulo $\pi$ ). This assumption does not restrict our study because the phase change on the reference surface cancels out when only the relative phase changes on the object 


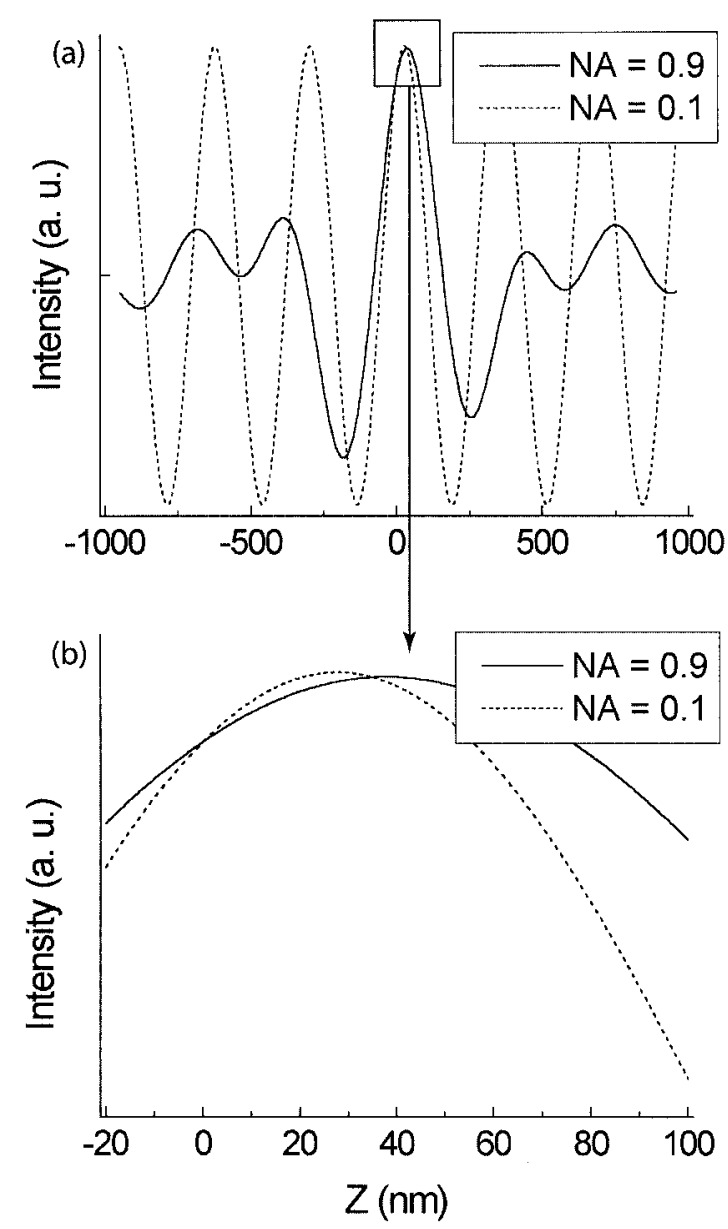

Fig. 2. (a) Numerical calculation of the interferogram response $M(z)$ with different numerical apertures [see Eq. (15)]. The object is a copper surface, with a phase change on reflection $\phi(\theta)$ calculated with Eq. (7). (b) Magnification of the peaks of both curves in (a) to show the shift of the interferogram with numerical aperture when the phase change on reflection is not zero.

surface (due to topography, phase change, or both, on reflection of dissimilar materials) are of interest. According to Eq. (15), the interferogram response $M(z)$ is the weighted sum of the elementary sinusoidal functions with weight, period, and phase depending on the incident illumination angle. As the illumination angle varies from $\theta=0$ to $\theta=\theta_{\max }$, the fringe spacing (the pseudoperiod) $\Lambda$ of $M(z)$ verifies

$$
\frac{1}{2 \sigma}<\Lambda<\frac{1}{2 \sigma \cos \theta_{\max }},
$$

and the position $z_{0}$ of the central fringe of $M(z)$ verifies

$$
\frac{-\phi(0)}{4 \pi \sigma}<z_{0}<\frac{-\phi\left(\theta_{\max }\right)}{4 \pi \sigma \cos \theta_{\max }} .
$$

We numerically calculated the function $M(z)$ by using the general formula in Eq. (7) for $\phi(\theta)$. The object was assumed to be copper because metallic materials (particularly copper) present a high phase change on reflection. ${ }^{8} M(z)$ is plotted in Fig. 2(a) with NAs of
Table 1. Theoretical Shift of the Central Fringe as a Function of Numerical Aperture for Copper at $\lambda=650 \mathrm{~nm}^{a}$

\begin{tabular}{ccccccccccc}
\hline \multicolumn{11}{c}{$\mathrm{NA}$} \\
\cline { 2 - 10 } & 0 & 0.1 & 0.2 & 0.3 & 0.4 & 0.5 & 0.6 & 0.7 & 0.8 & 0.9 \\
\hline
\end{tabular}

$\begin{array}{lllllllllll}\text { Shift }(\mathrm{nm})^{b} & 27.7 & 27.8 & 28.0 & 28.4 & 28.9 & 29.7 & 30.7 & 32.1 & 34.1 & 36.7\end{array}$

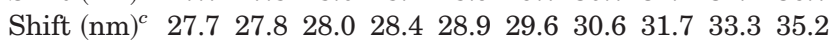
$\begin{array}{lllllllllll}\text { Shift }(\mathrm{nm})^{d} & 27.7 & 27.8 & 28.0 & 28.4 & 28.9 & 29.7 & 30.8 & 32.3 & 34.5 & 37.8\end{array}$ $\begin{array}{lllllllllll}\text { Shift }(\mathrm{nm})^{e} & 27.7 & 27.8 & 28.0 & 28.4 & 28.9 & 29.8 & 30.9 & 32.6 & 35.3 & 40.4\end{array}$

${ }^{a}$ The refractive indices of copper are $n=0.224$ and $k=3.63 .{ }^{14}$

${ }^{b}$ Obtained by numerical resolution of Eq. (15) with $\phi(\theta)$, calculated with Eq. (7) by use of the general Fresnel equations.

${ }^{c}$ Obtained by numerical resolution of Eq. (15) under the assumption that $\phi(\theta)=\phi(0)=-0.536 \mathrm{rad}(\operatorname{modulo} \pi)$.

${ }^{d}$ From Eq. (18) and the Taylor expansion of Eq. (15) to the third order in $\theta$.

${ }^{e}$ From Eq. (23).

0.1 and 0.9. $M(z)$ exhibits oscillations with an envelope that also oscillates and progressively decreases with $|z|$. The envelope narrows as the numerical aperture increases. The fringe spacing (the pseudoperiod of the oscillations) increases with the numerical aperture. The interferogram undergoes a shift that increases with the numerical aperture [see Fig. 2(b)]. The shift of the central fringe position is reported in Table $1^{b}$ for different numerical-aperture values.

To examine the influence of the dependence of $\phi$ on $\theta$ on the interferogram, we calculated $M(z)$ under the assumption that $\phi$ is constant and compared this result with that of the previous case in which $M(z)$ was calculated with $\phi$ dependence on $\theta$. The interferogram $M(z)$ is almost unchanged. The central fringe position undergoes almost the same shift with the numerical aperture (see Table $1^{c}$ ). The dependence of $\phi$ on $\theta$ therefore has almost no influence on the interferogram response of the interference microscope even at high numerical apertures. This result is in agreement with our demonstration in Section 3 that $\phi(\theta)$ is constant up to the third order in $\theta$.

The integral in Eq. (15) has already been calculated analytically under the assumption that the phase change $\phi$ is independent of the incidence angle $\theta .15$ Assuming a quadratic variation of $\phi$ with $\theta$, Eq. (15) can also be solved analytically by use of the small angle approximation. ${ }^{8}$ This approach is actually incorrect because $\phi(\theta)$ is constant up to the third order in $\theta$, at which point the modulated part of the interferogram expressed in Eq. (15) can be simplified to

$$
M(z)=V(z) \cos [\Phi(z)],
$$

with

$$
\begin{aligned}
V(z) & \propto \sin \left(\pi \sigma z \theta_{\max }^{2}\right) /\left(\pi \sigma z \theta_{\max }^{2}\right), \\
\Phi(z) & =4 \pi \sigma \alpha z+\phi(0) \\
\alpha & =1-\theta_{\max }^{2} / 4 .
\end{aligned}
$$

The interferogram response of the interference microscope can be regarded as a sinusoidal fringe pat- 
tern multiplied by an envelope $V(z)$, which has the shape of a $\sin (z) / z$ function. This result has already been shown elsewhere. ${ }^{15,16}$ It is justified rigorously here at the third order in the incidence angle. The $V(z)$ function is usually called the fringe visibility. The fringe spacing, equal to $1 / 2 \alpha \sigma$, depends on the numerical aperture through the factor $\alpha$, which is usually referred to as the aperture factor. This geometrical phenomenon has been widely studied. ${ }^{15,18-22}$ In our model the fringe spacing $\Lambda$ is equal to

$$
\Lambda=\frac{1}{2 \alpha \sigma}=\frac{1}{2\left(1-\theta_{\max }^{2} / 4\right) \sigma} .
$$

The position $z_{0}$ of the central fringe can be determined by numerical resolution of Eq. (18). The values are reported in Table $1^{d}$ and are in excellent agreement with the values found by numerical computation of Eq. (15), which confirms the validity of the model described by Eq. (18). An (approximate) analytical expression of the position of the central fringe would, however, be useful to see the influence of both $\phi(0)$ and the numerical aperture. At third order in the incidence angle, we obtain

$$
z_{0}=\frac{-\phi(0)}{4 \pi \alpha \sigma}=\frac{-\phi(0)}{4 \pi\left(1-\theta_{\max }^{2} / 4\right) \sigma} .
$$

When $\phi(0)=0$, the central fringe does not shift with NA $\left(z_{0}=0\right)$. When $\phi(0) \neq 0$, the central fringe undergoes a shift $z_{0}$, which increases nonlinearly with the numerical aperture. The values of this shift are calculated with Eq. (23) and are reported in Table $1^{e}$. They are close to those obtained previously by numerical computations until NA $<0.8$. The discrepancy for NA $>0.8$ is due to the effect of the envelope $V(z)$ that can no longer be considered constant around $z_{0}$.

\section{Measurements}

We present an experimental study of the influence of the phase change on reflection in interference microscopy as the numerical aperture is varied. We simultaneously measured the interferogram responses by using two different materials, a metal and a dielectric (glass), as objects placed in an interference microscope. For the metal we chose copper, which is known to exhibit a high phase change on reflection. To eliminate any phase change due to topography, the two materials must be planar and be at exactly the same height. To achieve this, we adopted the method proposed by Doi et al. ${ }^{6}$ The metal was evaporated on part of a microscope glass coverslip (0.17-mm thickness) by use of a mask. The metal film, with a thickness greater than $100 \mu \mathrm{m}$, was thick enough to be considered to have the same optical properties as a metal substrate. The experimental setup, depicted in Fig. 3, consists of a homemade Linnik interference microscope. ${ }^{15,16,23}$ We used identical slip-corrected microscope objectives (from Olympus) in both arms of the Linnik interferometer, with NAs varying from 0.1 to 0.9 . The object was

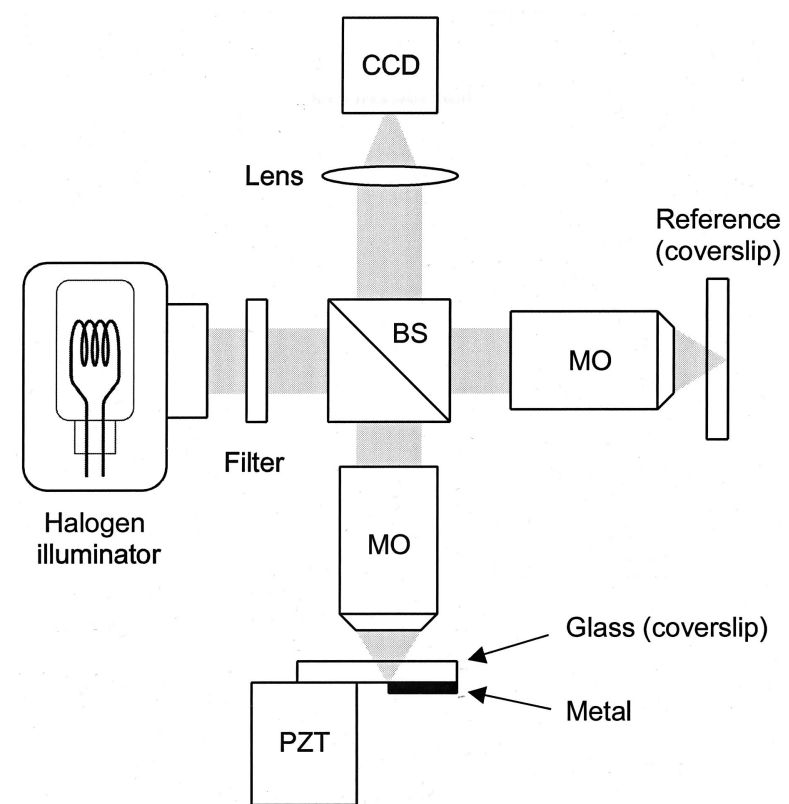

Fig. 3. Experimental setup: MO, coverslip-corrected microscope objectives (for various numerical apertures); BS, beam splitter; and PZT, piezoelectric transducer. The interference filter is centered at $\lambda=650 \mathrm{~nm}$, with a bandwidth $\Delta \lambda=10 \mathrm{~nm}$.

Table 2. Measurement of the Central Fringe Shift with Various Numerical Apertures for Copper at $\lambda=650 \mathrm{~nm}$

\begin{tabular}{ccccccc}
\hline & \multicolumn{6}{c}{ NA } \\
\cline { 2 - 7 } & $0.1^{*}$ & $0.25^{*}$ & 0.4 & 0.5 & 0.7 & 0.9 \\
\hline Shift $^{a}$ & 27.5 & 28.5 & 28.8 & 29.6 & 32.7 & 34.9 \\
Shift $^{b}$ & 27.8 & 28.2 & 28.9 & 29.7 & 32.1 & 36.7 \\
\hline
\end{tabular}

${ }^{a}$ Experimental.

${ }^{b}$ Theoretical (see Table 1, footnote $b$ ).

moved in the axial direction $(z)$ by means of a highprecision piezoelectric translation stage (Polytech PI Model P-753). The interferogram response was recorded in parallel by all pixels of the CCD camera (Dalsa CAD1). Some pixels corresponded to the image of the copper surface, others to the image of the glass surface. We measured the shift of the central fringe between the two interferograms (one corresponding to reflection on copper and the other to reflection on glass). The results, reported in Table 2, agree well with theory. The discrepancy, which is of the order of the absolute accuracy obtained in practice in phase-measuring interferometry $(\sim 1 \mathrm{~nm}),{ }^{1-3}$ includes the uncertainty in the numerical-aperture value marked on the objective barrel.

\section{Conclusion}

In profiling a surface composed of dissimilar materials by use of a phase-measuring interference microscope, the phase change on reflection is responsible for a shift of the interferograms, which leads to height measurement offsets. For accurate topographic measurements, these offsets must be corrected for by 
calculating or measuring the phase change on reflection. In this paper we have shown by analytical and numerical calculations that the phase change on reflection has a very weak dependence on the incidence angle (constant up to the third order). The phase change can be considered to be under normal incidence even when high numerical-aperture objectives are used. The shift of the interferogram due to the phase change on reflection increases with the numerical aperture. This results from an increase in the fringe spacing with the numerical aperture and, to a lesser extent, from a decrease in the fringe envelope width. This paper provides an approximate expression for the height offset correction to be applied [Eq. (23)], provided that the phase change (in normal incidence) and the numerical aperture are known. A more accurate compensating factor can be obtained by numerical evaluation of Eq. (18).

\section{References}

1. B. Bhushan, J. C. Wyant, and C. L. Koliopoulos, "Measurements of surface topography of magnetic tapes by Mirau interferometry," Appl. Opt. 24, 1489-1497 (1985).

2. F. Laeri and T. C. Strand, "Angström resolution optical profilometry for microscopic objects," Appl. Opt. 26, 2245-2249 (1987).

3. K. Creath, Progress in Optics XXVI, E. Wolf, ed. (Elsevier, New York, 1988).

4. J. M. Bennett, "Precise method for measuring the absolute phase change on reflection," J. Opt. Soc. Am. 54, 612-624 (1964).

5. T. McWaid, T. Vorburger, J. F. Song, and D. ChandlerHorowitz, "The effect of thin films on interferometric step height measurements," in Interferometry: Surface Characterization and Testing, K. Creath and J. E. Greivenkamp, eds., Proc. SPIE 1776, 2-13 (1992).

6. T. Doi, K. Toyoda, and Y. Tanimura, "Effect of phase changes on reflection and their wavelength dependence in optical profilometry," Appl. Opt. 36, 7157-7161 (1997).
7. K. Leonhardt, H. J. Jordan, and H. J. Tiziani, "Micro-ellipso height profilometry," Opt. Commun. 80, 205-209 (1991).

8. J. F. Biegen, "Determination of the phase change on reflection from two-beam interference," Opt. Lett. 19, 1690-1693 (1994).

9. S. Shatalin, R. Juskaitis, J. B. Tan, and T. Wilson, "Reflection conoscopy and microellipsometry of isotropic thin film structures," J. Microsc. 179, 241-252 (1995).

10. C. W. See, M. G. Somekh, and R. D. Holmes, "Scanning optical microellipsometer for pure surface profiling," Appl. Opt. 35, 6663-6668 (1996).

11. G. D. Feke, D. P. Snow, R. D. Grober, P. J. Groot, and L. Deck, "Interferometric back focal plane microellipsometry," Appl. Opt. 37, 1796-1802 (1998).

12. K. Leonhardt, U. Droste, and H. J. Tiziani, "Topometry for locally changing materials," Opt. Lett. 23, 1772-1774 (1998).

13. M. Born and E. Wolf, Principle of Optics, 6th ed. (Pergamon, New York, 1989).

14. E. D. Palik, ed., Handbook of Optical Constants of Solids (Academic, New York, 1985).

15. A. Dubois, J. Selb, L. Vabre, and A. C. Boccara, "Phase measurements with wide-aperture interferometers," Appl. Opt. 39, 2326-2331 (2000).

16. G. S. Kino and S. S. C. Chim, "Mirau correlation microscope," Appl. Opt. 29, 3775-3783 (1990).

17. F. C. Chang and G. S. Kino, "325-nm interference microscope," Appl. Opt. 37, 3471-3479 (1998).

18. H. Mykura and G. E. Rhead, "Errors in surface topography measurements with wide-aperture interference microscopies," J. Sci. Instrum. 40, 313-317 (1963).

19. C. J. R. Sheppard and H. J. Matthews, "Imaging in highaperture optical systems," J. Opt. Soc. A 4, 1354-1360 (1987).

20. J. F. Biegen, "Calibration requirements for Mirau and Linnik microscope interferometers,” Appl. Opt. 28, 1972-1974 (1989).

21. K. Creath, "Calibration of numerical aperture effects in interferometric microscope objectives," Appl. Opt. 28, 333-341 (1989).

22. G. Schulz and K.-E. Elssner, "Errors in phase-measurements interferometry with high numerical aperture," Appl. Opt. 30, 4500-4505 (1991).

23. A. Dubois, "Phase map measurements by interferometry with sinusoidal phase modulation and four integrating buckets," J. Opt. Soc. A. 18, 1972-1979 (2001). 